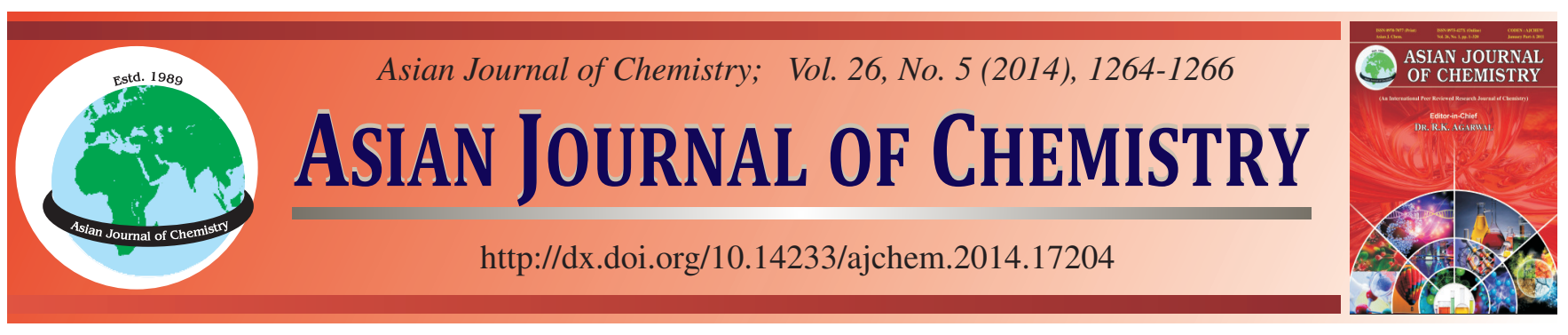

\title{
A Facile One-Pot Hydrothermal Method to Produce SnS/Reduced Graphene Oxide and Its Application in Removal of Dyes from Aqueous Solution $\dagger$
}

\author{
Lei Zhu, Sun-Bok Jo, Shu Ye, Kefayat Ullah, Ze-Da Meng and Won-Chun OH*
}

Department of Advanced Materials Science \& Engineering, Hanseo University, Seosan-si 356-706, Chungnam-do, Republic of Korea

*Corresponding author: Fax: +82 41 6883352; Tel: +82 41 6601337; E-mail: wc_oh@ @anseo.ac.kr

In this work, we report a novel one-pot synthesis of $\mathrm{SnS} /$ reduced graphene oxide nanocomposites via in situ reduction of graphene oxide by $\mathrm{Sn}^{2+}$ under ethylene glycol assisted hydrothermal conditions. The morphology and structure of the obtained product were characterized by Fourier transform infrared spectroscopy, X-ray diffraction, scanning electron microscopy with energy dispersive X-ray analysis, transmission electron microscopy and UV-visible diffuse reflectance spectrophotometer. The as-synthesized graphene-SnS composite efficiently catalyzed the photodegradation of methyl orange with high concentration in aqueous solutions under visible light irradiation. Based on the high photocatalytic activity, the graphene-SnS composite can be expected to be a practical visible light photocatalyst.

Keywords: Graphene, SnS, Visible light, Photocatalytic activity.

\section{INTRODUCTION}

The graphene-metal sulfide composites was traditionally prepared using graphene oxide as staring material, the oxygen containing groups in graphene oxide controlled the decoration of nanoparticles without aggregation. Besides, the reduction of graphene oxide led to graphene based composites with well charge. $\mathrm{SnS}$ is an orthogonal IV-VI group semiconductor material and has been proved to have potentially excellent properties for application in various areas, such as photovoltaic material ${ }^{1}$ etc. Jayalakshmi et al. ${ }^{2}$ investigated hydrothermally prepared nano $\mathrm{SnS}$ as an electrode active material for supercapacitor. Nano SnS electrode has good cycling stability and the capacitance is impressively higher in both alkali and neutral solutions. Peisong et al. ${ }^{3}$ reported synthesized SnS using a low cost and less toxic $\mathrm{SnCl}_{2}$ as the precursor and demonstrated that $\mathrm{SnS}$ nanomaterials possess high photocatalytic activity under visible light, which may be ascribed to the strong visible-light absorption and its efficient multiple exciton generation under the excitation of light irradiation with the higher energy than the band gap. However, up to date, less literatures report on the ethylene glycol assisted synthesis of graphene based $\mathrm{SnS}$ nanocomposite and applications for visible light photodecomposition of organic dye solution.

\section{EXPERIMENTAL}

Synthesis of graphene-SnS nanocomposites: In a typical experiment, about $20 \mathrm{mg}$ graphene oxide was dispersed in $100 \mathrm{~mL}$ ethylene glycol and then exfoliated to generate GONS dispersion solution by ultrasonication for $1 \mathrm{~h}$. Subsequently, $0.29 \mathrm{~g}$ of tin(II) chloride dihydrate powder was added to the GONS aqueous dispersion, followed by ultrasonication for $20 \mathrm{~min}$. Afterwards, anhydrous sodium thiosulfate and $10 \mathrm{~mL}$ ammonium hydroxide were added to the mixture under various stirring for $0.5 \mathrm{~h}$. which was in favour of the uniform distribution of SnS on GNS. After that, an amount of PVP powder was slowly added into the solution with vigorous stirring at $100{ }^{\circ} \mathrm{C}$ for another $4 \mathrm{~h}$. The final product was rinsed with distilled water and ethanol for several times and dried at $60^{\circ} \mathrm{C}$ for $12 \mathrm{~h}$.

Photocatalytic studies: In order to analysis of the photocatalytic effect, the degradation reaction of methylene orange in water was followed. For irradiation system, the visible light ( $\lambda>420 \mathrm{~nm}$, LED lamp) was used at the distance of $100 \mathrm{~mm}$ from the solution in darkness box, respectively. The clean transparent solution was analyzed by UV-visible spectroscopy. Details of methyl orange is given in Table-1. 


\begin{tabular}{c} 
TABLE-1 \\
MOLECULAR STRUCTURE AND ABSORBANCE \\
MAXIMUM $\left(\lambda_{\max }\right)$ OF METHYL ORANGE \\
\hline Organic dyes \\
Methyl orange
\end{tabular}

\section{RESULTS AND DISCUSSION}

Characterization: Fig. 1a is the illustration of deposition of $\mathrm{SnS}$ on the GONS. Fig. 1b shows the XRD pattern of the product obtained from the above procedure. It can be seen that the diffractogram of graphene exhibits the typical peaks at 25.9 and $42.7^{\circ}$, corresponding to the graphite (002) and (100) reflections (Joint Committee for Powder Diffraction Studies (JCPDS) No. 01-0646) ${ }^{4}$, respectively. In graphene-SnS composite all the diffraction peaks could be indexed to a simple cubic lattice with the cell constant $\mathrm{a}=4.3284 \AA$, that is consistent with the reported data for $\mathrm{SnS}^{5}$. However, no signal for any other phases about graphene oxide (001) or graphene (002) can be detected in graphene-SnS composite.
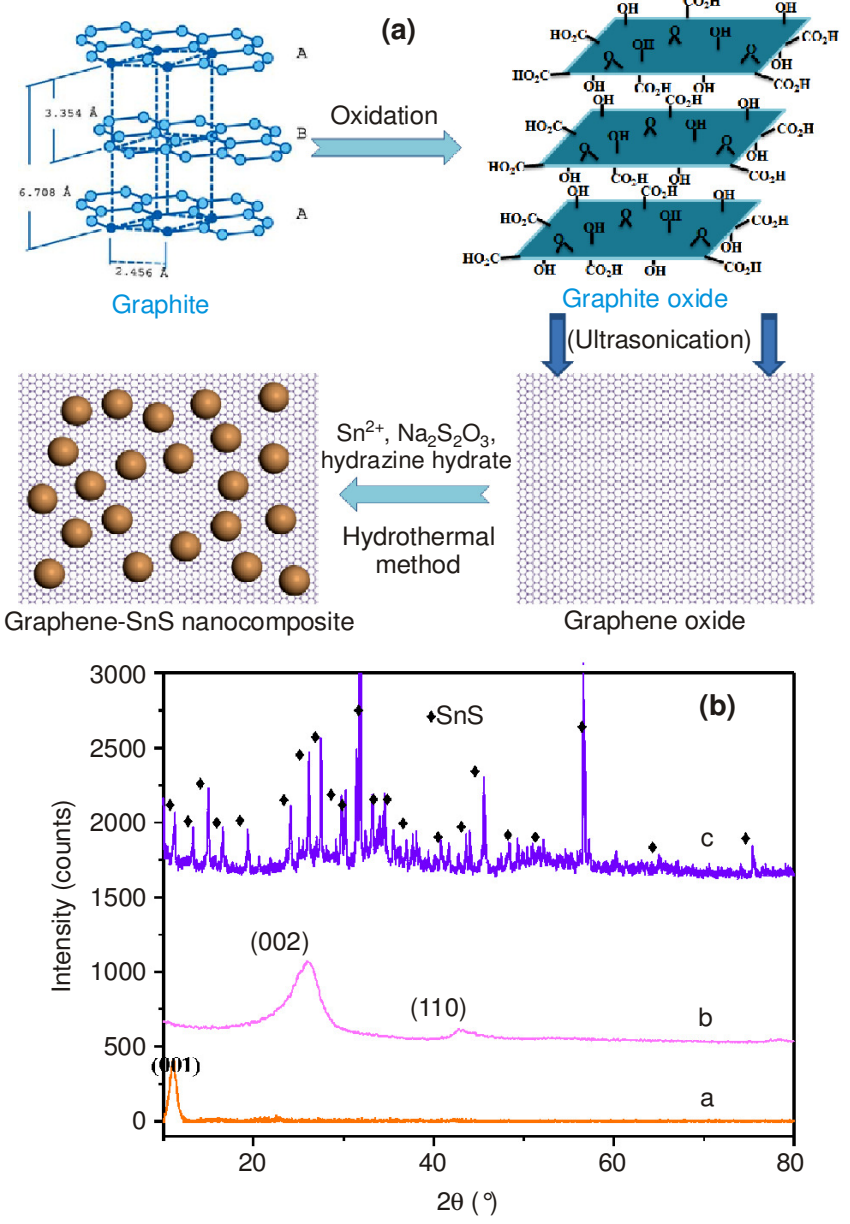

Fig. 1. (a) Schematic illustration of deposition of $\mathrm{SnS}$ on the graphene, (b) XRD pattern of graphene oxide, graphene and graphene-SnS composite

In Fig. 2a, it can be clearly seen that the pure graphene oxide sheets naturally aggregate and stack to multilayers with numerous edges. Moreover, the surface of graphene oxide was very smooth compared with graphene sheets doped with $\mathrm{SnS}$ nanoparticles in Fig. 2b. In Fig. 3, it could be seen that $\mathrm{SnS}$ nanospheres were uniformly distributed on a single layered graphene sheets, no apparent aggregation of the $\mathrm{SnS}$ spheres was discerned. The size of $\mathrm{SnS}$ spheres was calculated to be around 5-15 $\mathrm{nm}$.

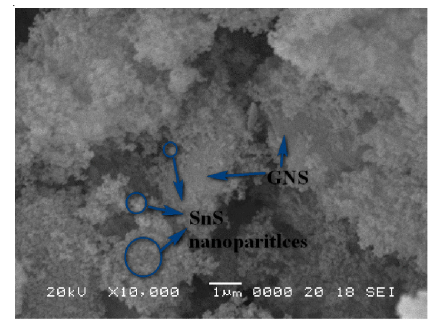

(a)

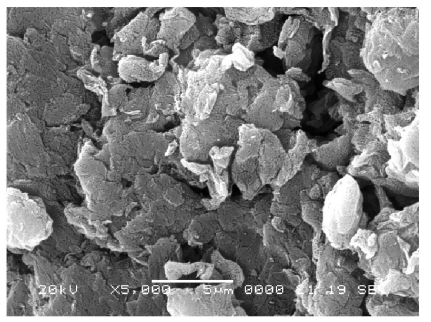

(b)
Fig. 2. SEM images of as-prepared composites: (a) graphene oxide, (b) graphene-SnS composite

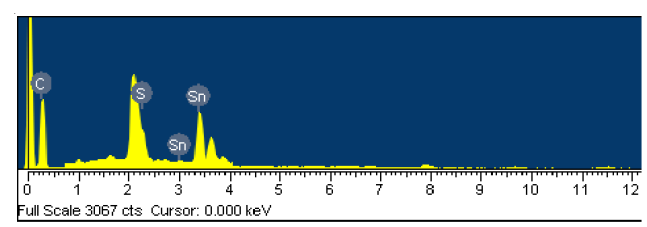

(a)

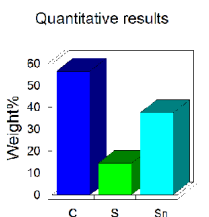

(b)
Fig. 3. EDX microanalysis (a) and element weight \% (b) of graphene-SnS composite

To get information about change in elements and element weight $\%$, the prepared graphene-SnS composite were examined by EDX. Fig. 3 shows the EDX microanalysis and element weight \% of graphene-SnS composite. Main elements such as $\mathrm{C}, \mathrm{Sn}$ and $\mathrm{S}$ are existed. The strong $\mathrm{C}$ signal should mainly originate from graphene nanosheets. The $\mathrm{Sn}$ and $\mathrm{S}$ peak comes from the precursor materials $\mathrm{SnCl}_{2} \cdot 2 \mathrm{H}_{2} \mathrm{O}$ and $\mathrm{Na}_{2} \mathrm{~S}_{2} \mathrm{O}_{3}$.

Photodecolorization process: The effect of catalyst composition on methyl orange $\left(3.00 \times 10^{-5} \mathrm{~mol} / \mathrm{L}, 100 \mathrm{~mL}\right)$ degradation efficiency was investigated under visible light irradiation with a catalyst amount of $0.03 \mathrm{~g}$. According to the results, a plot of $-\ln \left(\mathrm{c} / \mathrm{c}_{\mathrm{ads}}\right)$ versus $\mathrm{t}$ will yield a slope of $\mathrm{k}_{\mathrm{app}}$. The results are displayed in Fig. 4. The scheme of excitation and charge transfer process between SnS particles and graphene nanosheets under visible light irradiation is shown in Fig. 5.

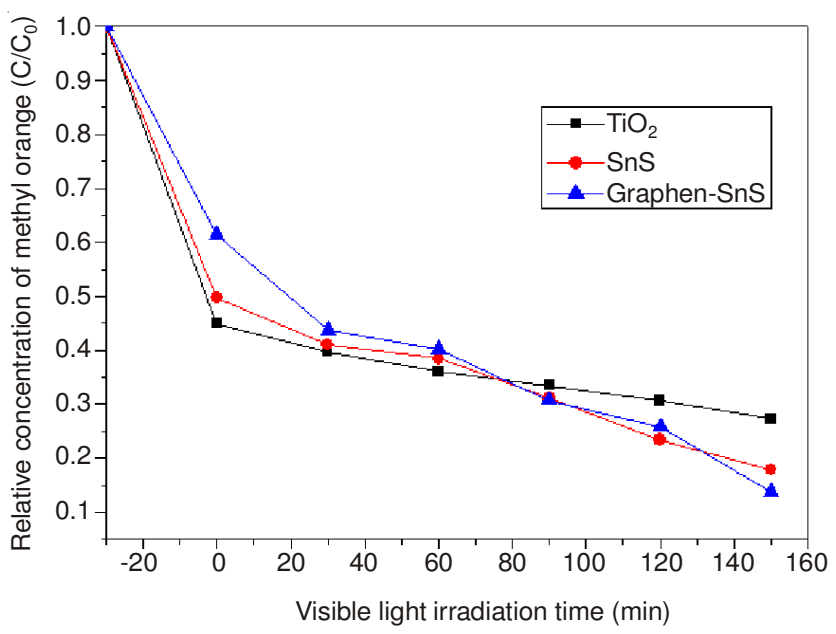




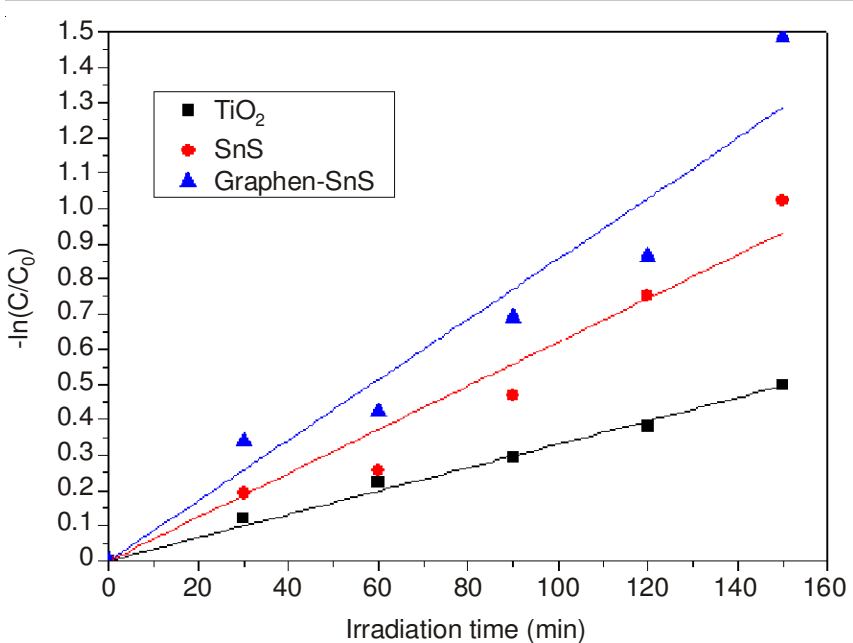

Fig. 4. Effect of the methyl orange decolorization process and the apparent first order kinetics of methyl orange in presence of $\mathrm{TiO}_{2}, \mathrm{SnS}$ and graphene-SnS photocatalysts

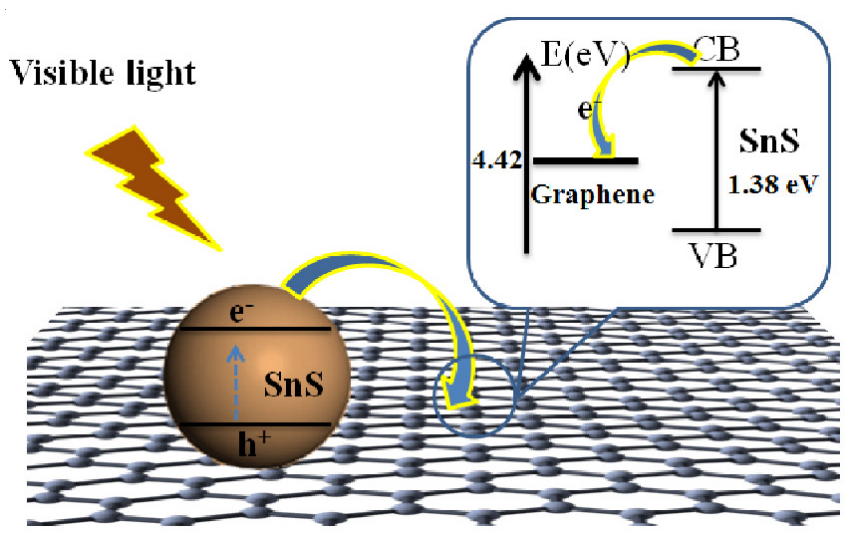

Fig. 5. Schematic diagram of the charge transfer between graphene and $\mathrm{SnS}$ under visible light irradiation

\section{Conclusion}

In this study, we report the obtention of graphene-SnS nanocomposites were successfully synthesized by a facile ethylene glycol assisted hydrothermal method. SEM and TEM observation showed that $\mathrm{SnS}$ nanoparticles were triggered by precipitation reaction uniformly distributed on the graphene sheets. The results reveal that graphene-SnS composite exhibit the highest photocatalytic activity on the degradation of methyl orange solution under visible light irradiation.

\section{REFERENCES}

1. N.K. Reddy and K.T.R. Reddy, Physica B, 368, 25 (2005).

2. M. Jayalakshmi, M. Mohan Rao and B.M. Choudary, Electrochem. Commun., 6, 1119 (2004).

3. P.S. Tang, H.F. Chen, F. Cao, G.X. Pan, K.Y. Wang, M.H. Xu and Y.H. Tong, Mater. Lett., 65, 450 (2011).

4. T. Ghosh, K.Y. Cho, K. Ullah, V. Nikam, C.-Y. Park and Z.D. Meng and W.C. Oh, J. Ind. Eng. Chem., 19, 797 (2013).

5. S.G. Hickey, C. Waurisch, B. Rellinghaus and A. Eychmüller, J. Am. Chem. Soc., 130, 14978 (2008). 